\title{
Observación y evaluación de los profesionales en los programas de apoyo a la parentalidad en la primera infancia: proceso de elaboración de un instrumento
}

\author{
Observation and evaluation of professionals in support programs \\ early childhood parenthood: process of an instrument
}

\author{
María FERRER RIBOT \\ Universidad de las Islas Baleares \\ maria.ferrer-ribot@uib.es \\ María Antonia RIERA JAUME \\ Universidad de las Islas Baleares \\ maria.riera@uib.es
}

Recibido: 02/04/2014

Revisado: 16/06/2014

Aceptado: 01/08/2014

Disponible on line: 10/12/2014

\begin{abstract}
Resumen
La Comisión Europea recomienda diseñar políticas para apoyar a los padres en el ejercicio de la parentalidad y ofrece directrices para los profesionales de los programas de apoyo e intervención familiar. En el artículo se presenta el proceso de elaboración de un instrumento diseñado para la observación y evaluación de los profesionales comprometidos con el trabajo social y que intervienen en dichos programas de atención a las familias en la primera infancia.

A partir de la revisión de los modelos teóricos y de un proceso de discusión con profesionales en ejercicio se describe el proceso de construcción y la estructura del instrumento. Los ítems están organizados en tres apartados: la organización del contexto, la intervención en las díadas padre/madre-hijo/hija y las actuaciones dirigidas al grupo de familias. A su vez cada uno de estos apartados incluye diversas dimensiones.

De la elaboración del instrumento se desprende que es fundamental diseñar herramientas que contribuyan a favorecer la reflexión y evaluación de los profesionales para realizar intervenciones ajustadas que aseguren la eficacia de los programas de intervención parental.
\end{abstract}

Palabras clave: instrumentos de observación y evaluación, primera infancia, programas de apoyo parental.

\begin{abstract}
The European Commission recommends designing policies to support parents in the exercise of parenting and provides guidelines for professional of support programs and family intervention. The article presents the process of developing an instrument designed for the observation and evaluation of professionals of Social Work involved in those programs serving families in early childhood.

From the review of theoretical models and a process of discussion with practitioners the paper described the construction process and the structure of the instrument. The items of the instrument are organized into three sections: the organization of context, intervention in dyads father / mother, son / daughter and group activities aimed at families. In turn each of these sections includes various dimensions.

The development of the instrument shows that it is essential to develop tools that help to promote reflection and professional assessment for interventions tailored to ensure the effectiveness of parental involvement programs.

Keywords: early childhood, observation and assessment tools, parental support, programs professional

Referencia normalizada: Ferrer Ribot, M. y Riera Jaume, M. A. (2014): «Observación y evaluación de los profesionales en los programas de apoyo a la parentalidad en la primera infancia: proceso de elaboración de un instrumento». Cuadernos de Trabajo Social, 27(2): 405-415.
\end{abstract}

Sumario: Introducción. 1. Método.

2. Proceso de construcción del instrumento. 3. Resultados. 4. Conclusiones. 5. Referencias bibliográficas. 


\section{Introducción}

A partir de los años 1980, ante los cambios sociodemográficos y las nuevas necesidades de las familias, surgieron programas de intervención con diferentes enfoques teóricos, por ejemplo, los desarrollados a partir de la aproximación «conductual», y los que se basaban en las relaciones interpersonales (Robles y Romero, 2011). Dichos programas se llevaron a cabo bajo diversas modalidades de intervención: grupal, individual, o combinados de ambos (Marinho y Ferreria, 2000) y entre sus objetivos estaban: fortalecer la vinculación afectiva entre padres e hijos, favorecer la creación de un clima familiar positivo, y acompañar a los padres en la crianza de los hijos.

Estas iniciativas siguen la Recomendación Europea (2006/19) del Comité de Ministros a los Estados miembros sobre políticas de apoyo al ejercicio positivo de la parentalidad, que recomiendan diseñar políticas encaminadas a ofrecer apoyo a los padres, creando vínculos sociales, promocionando grupos de ayuda y comunitarios, o manteniendo el apoyo formal desde los servicios profesionales. Algunos estudios (Menéndez, Hidalgo, Jiménez, Lorence y Sánchez, 2010) señalan la necesidad de programas psicoeducativos y experienciales que utilicen el formato de trabajo en grupo para fomentar redes de apoyo informales, especialmente dirigidos a familias en situación de riesgo psicosocial (Hidalgo, Menéndez, Sánchez, Lorence y Jiménez, 2007, 2009; Rodrigo, Máiquez, Martín y Byrne, 2008). En dichas recomendaciones se señalan también unas directrices para los profesionales tales como: reconocer la experiencia de los padres y el conocimiento sobre sus propios hijos, promover la iniciativa familiar, apoyar las fortalezas y recursos de las familias, entre otras. Las teorías sobre empowerment (Milani, 1997, 2008; Bouchard, 2002, Bouchard y Kalubi, 2003; Mendoza, 2004; Rodrigo, Máiquez, Martín y Byrne, 2008) conciben la figura del profesional como promotor de la parentalidad (partnership), partiendo de los recursos y competencias de las familias.

El Consejo de Europa destaca la importancia de prestar servicios a las familias a través de la formación de los profesionales, la evaluación y la continuidad de acción. También sugiere que se incluyan objetivos tan claros como dar respuesta a las necesidades de los niños y de los padres, o reconocer la importancia de que las familias compartan sus experiencias a través de metodologías grupales. En este sentido, parece que el formato grupal produce mejores resultados que otros, según Robles y Romero (2011), ya que ofrece a los padres la oportunidad de debatir y compartir experiencias con otras personas en similares circunstancias y con el apoyo de profesionales. Además esta formación es más apropiada en la relación entre costes y beneficios, resulta más atractiva que la intervención individual y ofrece un contexto adecuado para la práctica de las habilidades que implican la interacción social.

Aunque existen investigaciones sobre la eficacia y eficiencia de los programas de entrenamiento para padres (Martín, Máiquez, Rodrigo, Correa, y Rodríguez, 2004; Robles y Romero, 2011), encontramos todavía pocos estudios que tengan relación con la implementación y evaluación de programas en la primera infancia, algunos de ellos realizados en Italia (Milani, 2006a, 2006b) y en Bélgica (Vandenbroeck, 2010), en los que se analizan las expectativas de los padres sobre el apoyo a la parentalidad. Todos ellos coinciden en destacar el apoyo informal, basado en redes que favorezcan la diversidad de los grupos sociales.

También se constata la escasez de estudios sobre los profesionales y los modelos de intervención, algunos todavía de carácter exploratorio, como el de Gómez y Haz (2008), donde se abordan las teorías subjetivas de los profesionales que intervienen en programas de intervención familiar preventiva con familias multiproblemáticas. Las conclusiones de algunos estudios (Pourtois y Desmet, 2000; Pourtois y Desmet, 2006; Milani, 2006a, 2008) señalan la importancia de reflexionar sobre las tipologías de intervención de los profesionales, los criterios de eficacia, las condiciones de la calidad, las competencias y la formación necesaria de los profesionales en los servicios dirigidos a las familias.

En este artículo se presenta la elaboración de un instrumento que permite observar, reflexionar y evaluar las intervenciones y actuaciones que realizan los profesionales en los programas de apoyo a la parentalidad en la primera infancia. Entendemos que son necesarios unos instrumentos que ayuden a los profesionales a optimizar su intervención y sus recursos. Sin duda, 
la instauración de una práctica para evaluar las propias acciones favorecerá el avance del rol de los profesionales.

\section{Método}

\subsection{Sujetos}

El instrumento que se presenta se diseñó para aplicarlo a los profesionales que intervienen en los programas de apoyo a la parentalidad en la primera infancia, y está todavía en fase de experimentación, haciéndose ya algunos ensayos. Para la elaboración preliminar se ha contado con la colaboración del Grupo de investigación de primera infancia de la Universidad de las Islas Baleares, y de los 6 profesionales que trabajan en el Programa de Acompañamiento y Apoyo a Familias en la Primera Infancia (PAFPI), iniciado en Baleares en 2001, como recurso de apoyo a la parentalidad para familias en programas de reunificación o preservación familiar ${ }^{1}$. Las personas que trabajan en dicho programa provienen del ámbito de la pedagogía, psicología, magisterio de educación infantil y psicopedagogía, y su experiencia en el programa oscila entre los 4 y 8 años.

\section{Proceso de construcción del instrumento}

El instrumento está pensado para que se utilice en programas grupales con padres e hijos menores de 6 años, y que tengan como objetivos prioritarios: el fortalecimiento de la vinculación afectiva entre padres e hijos, el apoyo a la parentalidad en los primeros años de vida de los hijos, el refuerzo de las habilidades parentales y el favorecimiento del apoyo social mediante el trabajo en grupo. En dichos programas el rol del profesional se aproxima más a la figura de mediador que a la de experto (Cunningham y Davis,1998), facilitando las relaciones entre padres e hijos y favoreciendo las competencias y fortalezas de los padres desde la perspectiva de la parentalidad positiva (Consejo de Europa, 2006; Daly, 2007; Rodrigo, Máiquez y Martin, 2010, 2011).

\subsection{Fases para la construcción del instru- mento}

En una primera fase, con la ayuda de observadores externos, se realizaron 15 videograbaciones y se recogieron 30 registros de narraciones en las que se describían íntegramente todas las intervenciones de los profesionales durante las sesiones de trabajo con las familias.

En una segunda fase, se realizaron 3 grupos de discusión con los profesionales para analizar el material recogido e iniciar así una primera construcción de las dimensiones e ítems del instrumento. Se escogieron las intervenciones observadas consideradas positivas, entendiendo como positivas las que se ajustaban a los objetivos y características del programa.

En una tercera fase, se contrastaron los primeros ítems de acuerdo con los modelos teóricos explicativos de referencia que orientaban el programa, y que se exponen a continuación.

\subsection{Modelos de referencia teóricos}

Las perspectivas teóricas respecto a los aspectos contextuales y de relación que el profesional ha de contemplar, se vinculan con las dimensiones que recoge el instrumento.

\section{a) Teoría ecológico-sistémica: dimensiones contextuales}

La aportación de la perspectiva ecológica (Bronfenbrenner, 1987) señala la necesidad de contemplar los contextos de desarrollo del niño $\mathrm{y}$, concretamente, las interacciones sociales como elementos de protección. En este tipo de programas se mantiene la visión de los contextos como generadores de cambios en las conductas y en las relaciones humanas. Entendiendo por contexto el encuadre que organiza y ofrece una estructura al programa, el profesional debe diseñar, organizar y planificar las condiciones adecuadas para favorecer un contexto físico, temporal y relacional de calidad.

La orientación sistémica ofrece la visión de la familia como un sistema vivo y en proceso de transformación constante (Minuchin, 1986), y ayuda a entender las dinámicas de relación de la familia, así la atención se dirige a los sistemas, la comunicación y las pautas relacionales.

\section{b) Teoría del attachment: dimensiones de vinculación}

La teoría del apego o vínculo afectivo ofrece una visión relacional que ayudará a favorecer

El PAFPI se lleva a cabo por una asociación sin ánimo de lucro, Xarxa d'iniciatives d'Atenció a la Infancia i Families (XIAIF). 
contextos positivos de relación afectiva. La formación del sistema de apego (Bowlby, 1995) es el resultado de la interacción entre la actividad del niño que busca el contacto y la vinculación, y la actividad de los padres que le ofrecen un sistema de cuidado y protección (Winnicott, 1967; Ainsworth, Blehar, Waters y Wall, 1978).

Los afectos positivos y negativos y su regulación han sido estudiados en diversas investigaciones (Tronick, 1989; Brazelton y Cramer, 1993; Fonagy, Gergely, Jurist y Target, 2002). Destacamos también estudios realizados por Crittenden (1995), considerando la relación afectiva madre-hijo como un predictor del funcionamiento psicológico del niño.

Los padres sensibles, a través de la empatía conectan con las señales comunicativas de los hijos y responden adecuadamente, mientras que la falta de sensibilidad y empatía provoca a los hijos una inseguridad emocional. En este sentido, la sensibilidad de los padres se relaciona con la capacidad de leer e interpretar las señales del bebé, con la capacidad de atender y respetar la reciprocidad y saber mantener la espera en los diálogos interactivos (Ibáñez, 2005). Según la revisión de Escudero, Carranza y Huéscar (2013) del desarrollo de la atención conjunta en la infancia, la sensibilidad de los padres se manifiesta en conductas como: su habilidad para mantener la atención de su hijo hacia la tarea, la cantidad de tiempo que dedican a compartir la atención; así como la capacidad para simplificar y señalar sus características relevantes.

Algunos estudios (Díaz-Herrero, Pérez-López, Martínez-Fuentes, Herrera y Brito, 2000), muestran la necesidad de incluir, en los programas de intervención para padres, la comprensión del temperamento de los hijos, de modo que se le pueda ayudar a interpretar las señales de sus hijos y a evitar estimulaciones inadecuadas para optimizar su desarrollo, a través de algunos conceptos relacionados con la empatía: simetría/asimetría (Kaye, 1982; Brazelton y Cramer, 1993), intervenciones contingentes (Brazelton y Greenspan, 2005) e intersubjetividad (Trevarthen, 1982; Wertsch,1984; Yárnoz, 2006).

En el proceso de vinculación y regulación mutua entre padres e hijos, el profesional puede dar apoyo a las familias y favorecer modelos de vinculación positivos. Ofrecer un ambiente emocional afectivo, respetuoso y de aceptación a las personas por los profesionales, y disponer de capacidad para construir relaciones de confianza y seguridad y una escucha empática, son elementos clave para acompañar el proceso de construcción del rol parental.

\section{c) Teoría interaccionista: dimensiones de interacción}

Numerosos autores han diseñado estudios en que adultos y niños se ven comprometidos en una situación de actividad conjunta, de tipo diádico donde las relaciones de asimetría entre padres e hijos favorecen el desarrollo y aprendizaje de los niños (Wertsch, 1984, 1987; Rogoff, 1993; Méndez y Lacasa, 1995).

El concepto de participación guiada (Rogoff, 1993) es uno de los principios del desarrollo y aprendizaje del niño que explica el proceso en el que se entrelazan los papeles que desempeñan el niño y su cuidador, de manera que las interacciones y la forma en que se organiza la actividad proporcionan al niño oportunidades de aprendizaje, tanto implícitas como explícitas. El aprendizaje del niño se generará a través de la implicación de los adultos que apoyan, estimulan y fomentan las destrezas necesarias. Según Rogoff (2003) la «apropiación participativa», el aprendizaje, solamente es posible si antes hay «participación guiada». En este sentido, el profesional tiende puentes a las familias entre la información o las destrezas ya adquiridas y las que necesitan para resolver problemas nuevos en la interacción con sus hijos, realiza por tanto un proceso de participación guiada con las familias en relación a la parentalidad.

Destacamos también las aportaciones de Kaye (1982) que identifica siete tipos diferentes de marcos de interacción mediante los cuales los padres estructuran el mundo para sus hijos: el de protección, el instrumental, el de feedback, el de modelado o demostración, el de discurso y el de memoria. Los adultos (profesionales y padres) intentan encontrar estrategias para que el niño se implique: segmentan y dosifican la tarea; la organizan en metas y submetas alcanzables y manejables; proporcionan orientaciones, modelos, soportes, regulaciones, etc.; aumentan o disminuyen el nivel de exigencia o de ayuda; amplían, diversifican o reducen el contenido a compartir, ceden mayor o menor grado de protagonismo al niño o niña.

La función de andamiaje desarrollada por Bruner (1997), retomando las ideas de Vygotsky 
(1978) y la noción de zona de desarrollo próxi$m o$, introduce el concepto de «andamiaje», según el cual el adulto (padres y profesionales) «monta» o «sostiene» las consecuciones del niño, forzándole a entrar en la zona de desarrollo próximo y enseñándole a conseguir el control consciente de lo que va aprendiendo gracias a las relaciones sociales establecidas.

\section{d) Teoría de los grupos: dimensiones gru- pales}

En psicología social, una tarea, entre las otras fundamentales del profesional es conducir los grupos de familias, gestionar los conflictos y poseer habilidades comunicativas (Rogers, 1977; Belbin, 1993; Huici, 1996). En Morales y Olza (1996) encontramos descritas las tres formas de establecimiento de vínculos entre las personas, o de integración social, que se corresponderían con las tres funciones propias de los grupos, promoviendo así la integración ambiental, conductual y afectiva. Los programas que nos ocupan permiten a las familias compartir momentos y propuestas que variarán según la naturaleza del grupo y el tipo de integración que les caracterice. Así, por ejemplo, la integración afectiva de los miembros del grupo será un pilar básico ya que se constituye un tejido de relaciones, facilitador de conexiones e intercambios entre adultos, entre niños y adultos y entre niños y niños (Ferrer, 2008). A lo largo de los encuentros entre las familias se crean mecanismos de relación y se desarrollan sentimientos compartidos, indicadores del proceso individual realizado por cada familia y que se traducen en cambios importantes en la relación de los padres con sus hijos (Ferrer, Hernández y Ribas, 2009). El grupo se convierte en una herramienta potenciadora de cambio y permite crear vínculos entre sus miembros, ofrece ideas para solucionar problemas, proporciona identidad, compañía, aprendizaje, reconocimiento y sentimiento de pertenencia (Arija, 2012).

La cohesión es un concepto planteado por algunos autores como un pilar central en el estudio de los grupos (Saavedra, 1998) y se refiere al sentimiento de pertenecer a un grupo, al conjunto de fuerzas positivas y negativas que tiene como resultado que los integrantes permanezcan dentro del grupo. Si nos centramos en los programas grupales de intervención familiar, hay que considerar que los grupos de familias se forman de manera artificial y, por lo tanto, puede suceder que aunque los miembros sepan que pertenecen al grupo, no necesariamente se sientan incluidos. De esta manera el profesional ligado al ámbito social habrá de plantear situaciones que permitan aumentar la comunicación y la interacción, y promover la atracción social, partiendo del desarrollo de sentimientos de identidad y pertenencia entre las personas. El grado de cohesión influirá de manera directa en el rendimiento global del grupo, así como en el rendimiento individual de sus integrantes. Cuanto mayor sea el grado de unidad del grupo, mayor será la satisfacción de sus integrantes de pertenecer al mismo. Así, un liderazgo compartido, no directivo, la actitud empática, de escucha, de apoyo y de aceptación, y la estabilidad por parte de los profesionales favorecerán sentimientos de cohesión grupal.

Trabajar con grupos requiere especialmente a los profesionales vinculados al Trabajo Social una formación técnica, así como ciertas actitudes y características personales para actuar como un referente para el grupo y como agente de cambio. Algunas de estas características, señaladas por Castillo y Cabrerizo (2004), Barudy y Dantagnan (2005), Ferrer (2008), entre otros, son: estabilidad emocional, capacidad empática, capacidad de observación, capacidad de contención, actitudes de respeto y confianza, capacidad de autocrítica, creatividad, habilidades de trabajo en equipo y asertividad.

\section{Resultados}

Dado que el instrumento debe ser apropiado para el uso de los propios profesionales y de los observadores externos, se han considerado los siguientes factores:

a) Que sea fácilmente comprensible

b) Que recoja las intervenciones de los profesionales más relevantes en cada una de las dimensiones propuestas

c) Que sea breve, sencillo y fácil de administrar.

Para ello se ha elaborado en un formato de check-list en el que se recoge la presencia o ausencia del ítem que se evalúa.

Como resultado del proceso de revisión teórica y discusión con los profesionales que han colaborado en el diseño del instrumento, se pre- 


\begin{tabular}{|c|c|}
\hline Modelo teórico & Dimensiones del instrumento \\
\hline Teoría ecológico-sistémica & Dimensiones contextuales \\
\hline (Bronfenbrenner, 1987; Minuchin, 1986) & $\begin{array}{l}\text { Relacionadas con el diseño, organización y manejo de los } \\
\text { contextos físico ( } 9 \text { ítems), relacional (16 ítems) y temporal ( } 4 \\
\text { ítems) }\end{array}$ \\
\hline Teoría del attachment & Dimensiones de vinculación \\
\hline $\begin{array}{l}\text { (Bowlby, 1995; Ainsworth et al., 1978; } \\
\text { Belsky, 1999) }\end{array}$ & $\begin{array}{l}\text { Relacionadas con intervenciones que promueven contactos de } \\
\text { calidad y potencian la progresiva autonomía de los hijos (14 } \\
\text { ítems) }\end{array}$ \\
\hline Teoría interaccionista & Dimensiones de interacción \\
\hline $\begin{array}{l}\text { (Rogoff, 1993, 2003; Vygotski, 1978; } \\
\text { Bruner, 1997) }\end{array}$ & $\begin{array}{l}\text { Relacionadas con estrategias de actuación que potencian la } \\
\text { actividad conjunta entre padres e hijos y que favorecen la ob- } \\
\text { servación y la reflexión de los padres ( } 8 \text { ítems) }\end{array}$ \\
\hline Teoría de los grupos & Dimensiones grupales \\
\hline $\begin{array}{l}\text { (Lewin, 1978; Rogers, 1973; } \\
\text { Pichon-Riviere, 1995) }\end{array}$ & $\begin{array}{l}\text { Relacionadas con la construcción de una red social de apo- } \\
\text { yo y el fortalecimiento y cohesión del grupo ( } 9 \text { ítems) }\end{array}$ \\
\hline
\end{tabular}

Tabla 1. Modelos teóricos y dimensiones del instrumento.

senta a continuación la estructura del mismo organizado en 3 apartados, 6 dimensiones y un total 54 ítems.

Veamos a continuación algunos ejemplos de las intervenciones que se evalúan en cada uno de los apartados y dimensiones.

\section{Apartado 1: Contextos}

El primer apartado recoge las intervenciones de los profesionales en relación a los contextos, entendiendo que el contexto se convierte en el encuadre del programa y, por lo tanto, éste debe

\begin{tabular}{|l|}
\hline APARTADO 1: CONTEXTOS \\
\hline Dimensión 1. Contexto físico \\
\hline Dimensión 2. Contexto afectivo-relacional \\
\hline Dimensión 3. Contexto temporal \\
\hline APARTADO 2: DIADAS \\
\hline Dimensión 4. Vinculación \\
\hline Dimensión 5. Interacción \\
\hline APARTADO 3: GRUPO \\
\hline Dimensión 6. Grupal \\
\hline
\end{tabular}

Tabla 2. Estructura del instrumento. ser claro y estable. En este apartado se recogen las tres grandes dimensiones contextuales: física, relacional y temporal, organizadas en un total de 26 ítems.

La importancia de los contextos es crucial en este tipo de programas en los que se produce una articulación profunda entre el tiempo, el espacio y las relaciones. Así, se incluyen aspectos relacionados con el diseño y la organización del contexto físico, del contexto afectivo-relacional y del contexto temporal.

\section{Dimensión 1- Contexto físico}

Cuidar la calidad de los espacios y equipamientos contribuirá a generar sensaciones de bienestar y armonía, seguridad y confortabilidad. El entorno físico comunica sensaciones y actitudes a las personas que los habitan, provoca emociones, sugiere conductas de tranquilidad o, por el contrario, pueden resultar estresantes y caóticas (Riera, 2005). Los espacios y materiales de juego se convierten en mediadores de las relaciones entre padres e hijos, la observación del uso que hacen las familias será de gran utilidad para adaptar y modificar el espacio-ambiente a las necesidades y preferencias de cada una de ellas. En esta dimensión se incluyen algunos de los siguientes ítems: preparar el escenario de juego, cuidar la ambientación y estética, presentar a las 
familias nuevos espacios y materiales o sugerir nuevas maneras para su utilización.

\section{Dimensión 2 - Contexto afectivo-relacional}

Se incluyen aspectos relacionados con la capacidad del profesional para establecer relaciones empáticas y respetuosas con las familias y los niños: el modo cómo el profesional recibe $\mathrm{y}$ despide a las familias, la forma de relacionarse con ellas, el tono de voz y el vocabulario empleado, la distancia física, el acompañamiento a las familias nuevas, etc.

\section{Dimensión 3 - Contexto temporal}

El tiempo es un elemento, también, digno de consideración, ya que adquiere un importante significado la manera como se organiza y constituye una valiosa herramienta que dota de estructura al programa. Del mismo modo, la estabilidad en la estructura de las sesiones del grupo permite crear en las personas sentimientos de seguridad y control, a la vez que contribuye a transmitir una pauta de previsibilidad que asegura la tranquilidad y el conocimiento de lo que va a acontecer en cada momento del encuentro.

La regularidad y el equilibrio en las rutinas que se establecen favorecen la estabilidad en las relaciones, necesaria para asumir una buena parentalidad y un desarrollo sano en los niños. En este apartado se analizan algunos aspectos de las intervenciones del profesional, tales como: promover situaciones de tranquilidad entre padres e hijos o facilitar el cambio y las transiciones temporales, entre otros. Así, la anticipación a las familias de los diferentes momentos propicia una participación más activa por parte de los padres, y para los niños el conocimiento de la secuencia temporal, favorece un marco de seguridad, fundamental en las primeras edades.

\section{Apartado 2: Diadas}

El segundo apartado del instrumento recoge las intervenciones centradas en las relaciones de díada padres-hijos y en él se incluyen dos dimensiones: vinculación (13 ítems) e interacción (7 ítems), con un total de 20 ítems. Estos programas requieren realizar actuaciones dirigidas específicamente a cada una de las diadas. Bronfenbrenner (1987) señala que una diada se forma una vez que dos personas prestan aten- ción o participan cada una en las actividades de la otra, y según el potencial que tenga para aumentar el desarrollo psicológico. Hay diversas formas funcionales de díadas:

- Diada de observación, se produce cuando uno de los miembros presta atención a la actividad del otro.

- Diada de actividad conjunta, es aquella en la cual los dos participantes se perciben a sí mismos haciendo algo juntos.

\section{Dimensión 4 - Vinculación}

El profesional puede ofrecer modelos favorecedores de vínculos de calidad en la relación con los/as niños/as: relacionarse con gestos suaves y delicados, anticipar las acciones, favorecer la modulación del tono de voz o adaptar el vocabulario. Promueve también el contacto corporal, visual y verbal de los padres con sus hijos. Con el objetivo de favorecer la autonomía de los niños/as, el profesional acompaña el proceso de separación entre padres e hijos: ofrece modelos de conductas no intrusivas con los niños, promueve la espera y la no precipitación de actuación de los padres. Acompañar a las familias de un modo respetuoso obliga al profesional a controlar también su capacidad de regulación de la proximidad-distancia con las familias.

\section{Dimensión 5 - Interacción}

El profesional potencia la actividad conjunta entre padres e hijos e induce a los padres a la observación y la reflexión: segmenta y dosifica las tareas; proporciona orientaciones, modelos, soportes, regulaciones; cede mayor o menor grado de protagonismo al niño o niña; aumenta o disminuye el nivel de exigencia o de ayuda, etc.

En esta dimensión se incluyen tareas en las que se favorezcan situaciones donde se impliquen los padres y los hijos conjuntamente: proponer actividades lúdicas, facilitar la implicación de los padres en el juego y organizar momentos colectivos (rituales de entrada, de despedida, juegos, talleres, etc.).

Uno de los aspectos fundamentales es plantear situaciones donde los padres puedan conocer y observar a su hijo en un contexto distinto del familiar, lo que permitirá ajustar sus respuestas y desarrollar la capacidad empática. En este sentido, por ejemplo, el profesional puede ayudar a los padres verbalizando e interpretan- 
do las demandas y necesidades que observa de los niños o señalando los progresos y cambios evolutivos que se van desarrollando.

\section{Apartado 3: Grupo}

El tercer apartado del instrumento recoge las actuaciones de los profesionales dirigidas a la cohesión del grupo y al momento grupal con los padres como un espacio de aprendizaje y crecimiento (7 ítems). Los programas que contemplan la perspectiva grupal constituyen un elemento de apoyo fundamental para las familias en su labor de crianza y de cuidado hacia los hijos y contribuyen a la vez a incrementar la red social en la que se verán apoyadas. De este modo, se aprecia como el grupo puede llegar a convertirse en un espacio de aprendizaje, de crecimiento personal y de referencia social.

\section{Dimensión 6 - Grupal}

El profesional utiliza estrategias para favorecer la integración y participación de todos los miembros: contrastar experiencias y puntos de vista en relación a la paternidad, facilitar el diálogo y la conversación, recoger las necesidades y demandas, éstos son algunos de los ítems incluidos. Un elemento que ayuda a cohesionar el grupo es hablar y reflexionar sobre las propias actuaciones en relación a los hijos. De ahí que los profesionales escojan situaciones significativas de la sesión y en momentos de intercambio grupal se pongan como ejemplo de modelos de actuación.

También es una función de los profesionales proponer temas de debate relacionados con la crianza de los hijos: alimentación, sueño, autonomía, organización de los horarios, etc. Se trata de que todos participen en las conversaciones, favoreciendo la libertad de expresión, pero al mismo tiempo dando pautas para mejorar la dinámica del grupo (respetar los turnos de palabra, aprender a escuchar) y destacar las intervenciones que el profesional considere más relevantes para el grupo.

\section{Conclusiones}

Como se ha señalado, esta primera versión del instrumento ha sido objeto de discusión en el seno de un grupo focal formado por los profesionales del Programa de Acompañamiento y Apoyo a Familias en la Primera Infancia (PAFPI). Dichos profesionales valoraron positivamente la versión inicial del instrumento y contribuyeron con sus comentarios a la revisión del mismo. Aportaron también algunas sugerencias de cambio y de mejora acerca de la claridad y relevancia de los ítems:

a) Se incluyeron ítems repetidos o con un significado muy parecido.

b) Se propusieron cambios en la redacción y concreción de algunos ítems.

c) Se realizaron aportaciones para la incorporación de nuevos ítems.

Algunas de las valoraciones realizadas respecto a los beneficios de disponer de dicho instrumento fueron, entre otras: favorecer el autoconocimiento en relación a las estrategias utilizadas en las intervenciones, hacer consciente el propio estilo profesional y detectar las dificultades así como las fortalezas en la actuación profesional. En este sentido el instrumento puede utilizarse para la autoevaluación, la evaluación externa, así como para ofrecer formación y ayuda a los profesionales noveles. En cualquier caso, el objetivo fundamental es favorecer la reflexión de los propios profesionales para realizar intervenciones ajustadas y conseguir los objetivos de los programas.

Cabe señalar que los profesionales de diferentes ámbitos (trabajo social, educación, psicología, etc.) que trabajen con familias han de tener una formación específica y adecuada para responder a las diversas necesidades cambiantes de cada familia. Partiendo de este marco inicial, hay que considerar que la complejidad y la diversidad de intervenciones que implica el trabajo grupal con familias requiere contemplar, además, otros aspectos importantes: una formación inicial y continua, y conocimientos sobre el funcionamiento del sistema familiar, la teoría del apego, el maltrato infantil, psicología evolutiva, $\mathrm{y}$ estrategias de intervención en el trabajo con niños (Ferrer y Riera, 2007).

Haciendo referencia al perfil y características de los profesionales de la intervención social entendemos que es, en buena medida, su formación y estilo personal lo que contribuye a la calidad del programa. Algunos autores, entre ellos Barudy y Dantagnan (2005), recomiendan que los profesionales que trabajan con la infancia y familias desarrollen varias capacidades personales: la vinculación, la empatía y la sensibilidad. 
El trabajo de los profesionales debería basarse en la identificación y potenciación de las competencias parentales para favorecer, de este modo, su consolidación. Esta actuación rehúye del modelo de experto y especialista, siguiendo la clasificación de Cunningham y Davis (1998), que proporciona un saber especializado sobre la parentalidad. Defendemos, por lo tanto, un rol orientado hacia un profesional empático y sensible a las demandas y dificultades de las familias que favorece el intercambio y promueve el descubrimiento de las propias capacidades parentales.

La proliferación de programas de apoyo familiar obliga a repensar el perfil y la formación de los profesionales que intervienen, y a adoptar medidas que garanticen una atención de calidad a las familias que repercuta en el bienestar y desarrollo de los hijos. Se necesitan profesionales preparados y con un perfil adecuado para realizar un acompañamiento respetuoso y atento a las demandas de cada una de las familias, tal como señalan (Milani, 2006a; Pourtois, 2006).

La instauración de una práctica reflexiva que permita evaluar las acciones profesionales favorecerá, sin duda, la optimización de los programas. Es por ello por lo que disponer de instrumentos adecuados favorecerá el trabajo de reflexión de los profesionales comprometidos con la intervención social, contemplado como un proceso de transformación de la propia experiencia y convirtiendo al profesional en investigador en el contexto práctico.

\section{Referencias bibliográficas}

Ainsworth, M., Blehar, M., Waters, E. y Wall, S. (1978). Patterns of Attachment: A Psychological Study of the Strange Situation. New Jersey: Erlbaum.

Arija, B. (2012). Acompañamiento con grupos en Trabajo Social. Un modelo interfocal para la comprensión y sistematización de la praxis. Cuadernos de Trabajo Social, 25 (2), 27-438.

Barudy, J. y Dantagnan, M. (2005). Los buenos tratos a la infancia. Barcelona: Gedisa.

Belbin, M. R.(1993). Roles de equipo en el trabajo. Madrid: Belbin Associates.

Belsky, J. (1999). International and contextual determinants of attachment security. En J. Cassidy y P.R. Shaver (eds.), Handbook of Attachment: Theory, Research and Clinical Applications (pp. 249- 264). Nueva York: Guilford.

Bouchard, J.M. (2002). Partenariat et agir de communication: En: V. Guerdan, J.M. Bouchard y M. Mercier, Partenariat, chercheurs, praticiens, familles (pp. 77-87). Montreal: Les Éditions Logiques.

Bouchard, J.M. y Kalubi, J.C. (2003). Relazioni tra famiglia e professionisti: un passaggio obbligato o un tranello? En: L'animatore di educazione familiare: una nuova figura professionale? (pp. 83-96). Firenze: Istituto degli Innocenti, Regione Toscana.

Bowlby, J. (1995). Una base segura. Barcelona: Paidós.

Brazelton, T.B. y Cramer, B.G. (1993). La relación más temprana. Padres, bebé y el drama del apego inicial. Barcelona: Paidós.

Brazelton, T.B. y Greenspan, S. I. (2005). Las necesidades básicas de la infancia. Barcelona: Graó.

Bronfenbrenner, U. (1987). La ecología del desarrollo humano. Barcelona: Paidós.

Bruner, J. (1997). La educación, puerta de la cultura. Madrid: Visor.

Castillo, S. y Cabrerizo, J. (2004). Evaluación de programas de intervención soioeducativa: agente y ámbitos. Madrid: Pearson.

Crittenden, P. (1995). Attachment at risk for psychopathology: the early years. Developmental and Behavioral Pediatrics, 16 (3), 12-16.

Cunningham, C. y Davis, H. (1998). Trabajar con los padres. Marcos de colaboración. Madrid: Siglo XXI.

Daly, M. (ed.).(2007). Parenting in contemporary Europe: Apositive approach. Strasbourg: Council of Europe Publishing.

Díaz-Herrero, A., Pérez-López, J., Martínez-Fuentes, M.T., Herrera, E. y Brito, A. (2000). Influencias de la personalidad materna sobre el estilo conductual infantil: implicaciones para la atención temprana. Anales de Psicología, 16 (1), 101-110.

Escudero A., Carranza, J.A. y Huéscar, E. (2013). Aparición y desarrollo de la atención conjunta en la infancia. Anales de Psicología, 29 (2), 404-412. 
Ferrer, M. y Riera, M.A. (2007). El rol i les intervencions dels professionals en programes socioeducatius per a famílies de risc. Rev. Enginy. 16, 31-40.

Ferrer, M. (2008). Suport a les famílies en la primera infància. Estudi de cas d'un programa socioeducatiu. Tesis doctoral. Universitat de les Illes Balears. [http://www.tesisenred.net/handle/10803/9393]

Ferrer, M., Hernández, M. y Ribas, A. (2009). El programa Espai Familiar: la perspectiva relacional a partir de la intervención grupal. Revista Mosaico. FEATF, 43, 43-48.

Ferrer, M. (2010). Los programas de intervención familiar y de apoyo a la parentalidad. Indivisa, Bol. Estud. Invest, 11, 39-56.

Fonagy, P., Gergely, G., Jurist, E. y Target, M. (2002). Affect Regulation, Mentalilization: Developmental, Clinical and Theoretical Perspectives. Nueva York: Other Press.

Gómez, E. y Haz, A.M. (2008). Intervención familiar preventiva en programas colaboradores del SENAME: La perspectiva del profesional. Psykhe, 17, (2), 53-65.

Hidalgo, M.V., Menéndez, S. Sánchez, J., López, I. y Lorence, B. y Jiménez, L. (2007). Programa de Formación y Apoyo Familiar (FAF). Sevilla: Delegación de Bienestar Social del Ayuntamiento de Sevilla.

Hidalgo, M.V., Menéndez, S., Sánchez, J., Lorence, B. y Jiménez, L. (2009). La intervención con familias en situación de riesgo psicosocial. Aportaciones desde un enfoque psicoeducativo. Apuntes de Psicología, 27 (2-3), 413-426.

Huici, C. (1996). Las relaciones entre grupos. En J. F. Morales y M. Olza, (coords.). Psicología Social y trabajo social. Madrid: McGrawHill.

Ibáñez, M. (2005). Nuevas alternativas en investigación sobre apego e intersubjetividad. Instrumentos de evaluación. (Ponencia presentada en IV Jornadas Apego y Salud mental. International Attachment Network-Iberoamericana. Alicante).

Kaye, K. (1982). La vida mental y social del bebé. Barcelona: Paidós.

Lewin, K. (1978). La teoría de campo en la ciencia social. Madrid: Paidós.

Marinho, M.L. y Ferreira, E. (2000). Evaluación de la eficacia de un programa de entrenamiento de padres en grupo. Psicología Conductual, 8 (2), 299-318.

Martín, J.C., Máiquez, M.L., Rodrigo, M.J., Correa, A.D. y Rodríguez, G. (2004). Evaluación del programa «Apoyo personal y familiar» para madres y padres en situación de riesgo psicosocial. Infancia y Aprendizaje, 27 (4), 437-445.

Martín, J.C. Máiquez, M.L. Rodrigo, M.J. Byrne, S. Rodríguez, B. y Rodríguez, G. (2009). Programas de Educación Parental. Intervención Psicosocial, 18 (2), 121-133.

Méndez, L.y Lacasa, P. (1995). Aprender y enseñar en situaciones cotidianas: observando la interacción de Teresa con los adultos. En: P. Fernández-Berrocal y A. Melero (comps.), La interacción en contextos educativos (pp. 327-377). Madrid: Siglo XXI.

Menéndez, S., Hidalgo, M.V., Jiménez, L., Lorence, B. y Sánchez, J. (2010). Perfil psicosocial de familias en situación de riesgo. Un estudio de necesidades con usuarias de los Servicios Sociales Comunitarios por razones de preservación familiar. Anales de Psicología, 26 (2), 378-389.

Mendoza, R. (2004). Proyecto docente e investigador en trastornos del desarrollo. (Memoria de Cátedra inédita). Universidad de Huelva.

Milani, P. (1997). Empowerment. En Studium educationis, 6, 1043-1045.

Milani P.(2006a). L'aiuto informale tra famiglie: ragioni ed esperienze. En: R. Maurizio, F. Belletti y P. Milani (eds.), (pp. 30-59). Padua: Fondazione Zancan.

Milani P.y Pegoraro E. (2006b).Una carta ética nei servizi dell'infanzia? Una micro-ricerca fral'esistente e il possibile. En M. Manetti (ed.). Le ricerche per l'infanzia, 121-150. Bergamo: Junior.

Milani P., Gioga, G. y Frosi A., I (2008). Risultati della ricerca «Empowernet». En: G.Yugo, Famiglie, disabilità e servizi: parlano I protagonisti (pp. 30-112). Padua: Fondazione Zancan.

Minuchin, S. (1986). Familias y terapia familiar. Buenos Aires: Gedisa.

Morales, J. F. y Olza, M. (coords.). (1996). Psicología Social y trabajo social. Madrid: McGrawHill. Pichon Riviere, E. (1995). El proceso grupal. Del psicoanálisis a la psicología social.Buenos Aires: Nueva Visión. 
Pourtois, J.P. y Desmet, H. (2000). L'education familial comme intervention sociale. (Investigaciónacción realizada con la colaboración de los CPAS.Université de Mons-Hainaut, CERS).

Pourtois, J.P. y Desmet, H. (2006). La educación implícita: socialización e individualización. Madrid: Popular.

Riera, M. A. (2005). El espacio-ambiente en las escuelas de Reggio Emilia. Indivisa. Boletín de Estudios e Investigación, 3, 27-37.

Robles, Z. y Romero, E. (2011). Programas de entrenamiento para padres de niños con problemas de conducta: una revisión de su eficacia. Anales de Psicología, 27 (1), 86-101.

Rodrigo, M.J., Máiquez, M.L., Martín, J.C. y Byrne, S. (2008). Preservación familiar. Un enfoque positivo para la intervención con familias. Madrid: Pirámide.

Rodrigo, M.J., Máiquez, M.L. y Martín, J.C. (2010). Parentalidad positiva y políticas locales de apoyo a las familias. Orientaciones para favorecer el ejercicio de las responsabilidades parentales desde las corporaciones locales. Madrid: Federación Española de Municipios y Provincias (FEMP). Gobierno de España. Ministerio de Sanidad, Política Social e Igualdad.

Rodrigo, M.J., Máiquez, M. L. y Martin, J.C. (2011). Buenas prácticas profesionales para el apoyo a la parentalidad positiva. Madrid: Federación Española de Municipios y Provincias (FEMP). Gobierno de España. Ministerio de Sanidad, Política Social e Igualdad.

Rogers, C. (1973). Grupos de encuentro. Buenos Aires: Ed. Amorrortu.

Rogers, C. (1977). Psicoterapia centrada en el cliente. Buenos Aires: Paidós.

Rogoff, B. (1993). Aprendices del pensamiento. Barcelona: Paidós.

Rogoff, B. (2003). The Cultural Nature of Human Development. Londres: Oxford Universiy Press. Saavedra, I. (1998). Motivación y comunicación en las relaciones laborales. Madrid: Pirámide.

Trevarthen, V. (1982). Los motivos primordiales para entenderse y para cooperar. En: A. Perinat, La comunicación no verbal (pp.143-182). Barcelona: Avesta.

Tronick, E.Z. (1989). Emotions and emotional communication in infants. American Psychologist, 44, 112-119.

Vandenbroeck, M. (2010). Le soutien social de la parentalité en context urbain: faisabilité et limites. Università di Gand (Belgio). Conferencia presentada en el XIII Congresso Internazionale AIFREF. Firenze.

Vygotsky, L. S. (1978). Mind in Society: The Development of Higher Psychological Processes. Cambridge, MA: Harvard University Press.

Wertsch, J.V. (1984). The creation of context in joint problem solving. En: L. Rogoff, (ed.), Everyday cognition: its development in social context (pp.151-171). Cambridge: Harvard University Press.

Wertsch, J.V. (1987). Problem solving in social interaction: a microgenetic analysis. En M. Hickman (ed.), Social and functional approaches to language and thought (pp. 251-266). Orlando, Fl.: Academic Press.

Winnicott, D. (1967). La familia en el desarrollo del individuo. Buenos Aires: Paidós.

Yárnoz, S. (2006). Estilos de apego y regulación emocional. Ponencia presentada en $V$ Jornadas de Apego y Salud mental. International Attachment Network-Iberoamericana, San Sebastián. 